\title{
Implication of Post Production Defects in Software Industries
}

\author{
Divakar Harekal \\ Research Scholar Dept. of Computer Science \\ Engg., JJTU, Rajasthan
}

\author{
Suma V \\ Dayanandsagar Research and Industry \\ Incubation Centre, Dayananda Sagar Institutions, \\ Bangalore,India
}

\begin{abstract}
Development of defect free software is one of the primary concerns of any software industry. The main objective is to ship a high quality software product to their customers in order to attain total customer satisfaction. This is the only strategy through which industry can continue to sustain in the dynamic market. Hence, several defect management techniques are followed to reduce the defect leakage count during their production cycle. Nevertheless, the efforts laid by expert professionals in unearthing the maximum defects, yet there still prevails unidentified defects entering the customer's site. Unawareness to post production defects results in rework and overheads which is not an appreciable solution to the industry. The aim of this paper is therefore to investigate the implication of post production defects in software industries. This understanding facilitates the developing team to overcome the rate of defect injection during the production span such that post production defect leakage is reduced.
\end{abstract}

\section{Keywords}

Component, Software Engineering, Software Development Life Cycle, Post Production Defects, Defect Management, Software Quality, Software Process.

\section{INTRODUCTION}

The wide spread applications of software in every walk of life has made it a compulsion to develop, deliver and manage an effective software product. Failure of which, leads to undesirable and non acceptance of the software product by the stakeholders. Hence, every software production aims to develop projects having total customer satisfaction. Total customer satisfaction can be further achieved through high quality software. However, there are various perspectives in which quality is viewed by the customers. It includes quality means conformance to specified requirements. Quality also means development of the product within estimated budget, time, and available resources. Quality further applies to generation of products having zeroed or minimal defects

It is however worth to recall that complexity of problem domain indicates the existence of unavoidable intricacy in the software. They are due to contradicting requirements, impedance mismatch between system users and system developers, changing requirements, heavy capital investment in software etc. that inevitably makes the system to co-exist with the legacy systems.

Grady Booch adds up due to the difficulty in managing the development process. Development team requires effective coordination and co-operation among the team members. He further suggests that the developing team demands maintenance of unity and integrity of the design, regardless of geographically dispersed team [1].
Booch further expresses that due to the inherent complexity of software, it becomes difficult for the individual developer to understand the intricacies of its design. He concludes stating that it is possible to master the complexity but cannot avoid them[2].

Hence, software is prone to get developed with defects. However, defects if undetected in the preproduction cycle may only result in defect occurrences in post production cycle.

In 1989, Humphrey stated that quality is measured in terms of defects and software development process should be capable of fulfilling the needs of the customer with less cost. Further, he described from the theory of Personal Software Process (PSP), defects can be captured close to their origin through personal reviews and thereby reduce the cost for detection [3]. Hence, detection of defects early in the software development process put off the possibility of defect passage from requirement engineering phase to design or propagation of defects from design phase into code construction phase [4]. Additionally, defect detection in the right time and at the right phase enables the attainment of quality in terms of ensured reliability, maintainability, efficiency, portability and other such quality attributes [5].

Failure of defect capturing during pre production time of the software certainly leads towards defect leakage. This part of the research therefore aims towards analyzing the implications of defects that sneaked into the customer's site. The knowledge of this implication acts as a travel light for the developing team to enhance their detection skills and orient towards reduction of defect leakage.

\section{LITERATURE SURVEY}

All Wherever From the time when the Software Engineering significance is gained, every software industry has inclined towards development of qualitative software. In order to achieve this goal, various researchers have put forth their thoughts and ideas to improve the quality of software production.

Further, authors of [6][7][8] has aimed their research to investigate the prediction ability of success of the projects using advanced mining techniques such as Fuzzy logic, K means Clustering and Genetic Algorithms. Their investigations have indicated the dire need to improve the success level of projects through effective defect prediction techniques [6][7][8].

Hence, authors of paper [9][10][[11][12] have introduced two qualitative metrics, Depth of Inspection and Inspection Performance Metric to measure the depth of effectiveness of one of the software preventive techniques namely inspections. This introduction not only evaluates, but also estimates and 
predicts the efficiency and effectiveness of inspection process and team effort. Their work enables the software team to comprehend their efficacy of pre production defect capturing ability [9][10][[11][12].

Authors of [13][14] has proven the need to enhance software testing techniques. They have investigated the degree of testing that happens at every phase of software development. They have conducted a horizontal analysis of testing across the projects and vertical analysis of testing within individual projects [13][14].

Therefore, authors of [15][16] has worked to explore the significance of exploratory testing to capture more number of pre production defects such that defect leakage is reduced. Their work has proven the vitality of improving testing techniques [15][16]

Jef Jacobs et al.,] suggested the need for reducing residual defects. The objective of their study was to identify the factors that influences defect injection and defect detection to reduce residual defects. The results show the existence of several factors that influence defect detection such as testability, which comprises of test facilities, integrated in the product, the degree to which testability of the product has been included in the design and so on [17].

Effective defect removal reduces the developmental cost, time and enhances the product quality. Better defect prevention and removal activities maximize the effectiveness of the product. Nevertheless, software industries persist to deliver products with residual defects regardless of the following a number of defect management strategies. Factors such as state-of-the-art practice in defect management strategies, requirement for continuous quality improvement and significance of effective defect detection and prevention in software development motivated this search for the comprehending the implications of post production defects

\section{RESEARCH METHODOLOGY}

The main intention of this part of the research is to explore the impact of post production defects towards attainment of total customer satisfaction. Hence, this research initially started with identification of software industries for the investigation purpose. Based on selective sampling technique, software industries which are operating at CMMI Level 5 is selected. The rationale behind this selection is existence of effective defect management techniques to reduce post production defects. Further, projects developed for non critical applications are deliberately selected for this study since critical applications will hardly have post production defects. Thus, empirical investigations of projects which are developed in telecom domain since 2013 onwards to 2014 are considered.

The data for these projects are collected by the defect log of defect prevention and quality assurance department of the sampled industries. Having obtained the data, an analysis is initially carried out to investigate the implications of post production defects in the customer satisfaction level. This analysis has further driven towards exploring the existence of defect pattern and their root cause analysis, which are our forthcoming works.

\section{CASE STUDY}

Before The objective of this part of the research is to discover the connotation of existence of post production defects in software industries. This paper however, limits to present a case study carried out in one of the sampled leading software industry. The projects which are presented in Table 1 is developed in telecom domain using Java Programming language and J2EE. They are operating in windows and further uses the RFT (Rational Functional Test) tool, which is a test automation tool.

Table 1. thus presents a sample of 6 projects which are drawn from varying project complexity. These projects are having a complexity between 3 and 4 indicating that a complexity level of 1 is simple project and level 5 complexity in projects indicate the highest degree of complexity since in this set of samples from the industry, complexity is measured in a scale of 1 to 5. The table further provides information about defects escape, customer reported defects, time and cost of rework.

Defects escape is an in process defect, which is reported during User Acceptance testing. The software products having these defects are not yet shipped to the field and are still in the shop floor. However, customer reported defects are Post production defects which are identified in the software product which is deployed in the field and is identified by the customer during operational phase. Hence, a customer reported defect is taken up for fixing in the company for which the time and cost required is deemed as rework time and cost to fix that defect. The rework time and cost is proportional to the following factors:

- Defect Complexity and effect of regression

- Number of customer reported defects

- $\quad$ Number of in process defects

- System down time in case of post production defects

- Time to fix, retest and code drop

Table 1 clearly infers that Project 4 and Project 5 has the same level of complexity. However, in Project 5, since customer reported defects are comparatively more than other projects, customer satisfaction index has reduced. However, when looked from the perspective of rework time and cost also Project 5 has shown an increase due to increased number of customer reported bugs. It is also interesting to note that Projects P1 and P3 have same complexity, and customer reported defects are also same while rework time and cost has increased. The observation thus drawn to this information has driven this research to explore the nature of defect identified and root cause analysis for the same. However, this analysis will appear as our forthcoming work. Hence, as a limitation of this paper, it is intended to bring in awareness on the impact of post production defects and hence the orientation of the industry towards formulation of strategy to reduce the same 
(*)- Measured in person hours; (**) Thousand US Dollars

Table1. Customer Reported Defect Count

\begin{tabular}{|c|c|c|c|c|c|}
\hline Parameters & Project-1 & Project-2 & Project-3 & Project-4 & Project-5 \\
\hline $\begin{array}{l}\text { Project hours of } \\
\text { development }(*)\end{array}$ & 1390 & 1890 & 1460 & 2850 & 3440 \\
\hline Cost $(* *)$ & 1400 & 2100 & 1800 & 2900 & 3200 \\
\hline $\begin{array}{l}\text { Complexity (to the scale } \\
\text { of } 1 \text { to } 5 \text { ) }\end{array}$ & 3 & 3 & 3 & 4 & 4 \\
\hline \# of defects captured & 72 & 91 & 79 & 92 & 99 \\
\hline \# of escapes & 2 & 4 & 4 & 5 & 5 \\
\hline $\begin{array}{l}\text { \# of customer reported } \\
\text { defects }\end{array}$ & 1 & 2 & 1 & 2 & 3 \\
\hline $\begin{array}{l}\text { \# customer satisfaction } \\
\text { index(CSI) }\end{array}$ & 9.2 & 9.2 & 9.1 & 9.2 & 8.9 \\
\hline Rework Cost $(* *)$ & 300 & 550 & 400 & 600 & 670 \\
\hline Rework Time $(*)$ & 12 & 22 & 18 & 24 & 27 \\
\hline
\end{tabular}

\section{CONCLUSION}

After Since, software has laid its impact on every field of operation, development of customer satisfied software is the prime hour of the day. In view of the fact that, defect is one of the major contributing factor to retain customer satisfaction. Hence, this paper illustrates a case study of a sampled software industry where projects are investigated to unearth the consequences of defects in the production cycle. This part of research is to explore the impact of post production defects in the company. The in process defects are lot simpler to work on and close before the final code drop happens. This reduces costs and down time and has a significant bearing on Customer satisfaction. Post production defects may cause system down time and expensive to fix. The rework costs show a remarkable increase when there are more field defects, resulting in down time and reduced customer satisfaction values. However, the fixing time is also dependent on defect complexity, severity and regression effects. This awareness has yielded our forthcoming research to expose the defect modulating parameters and their root cause analysis in order to reduce the post production defect count

\section{ACKNOWLEDGMENTS}

The authors would like to sincerely acknowledge all the industry personnel who have helped in providing information and data support under the non disclosure agreement to carry out this research

\section{REFERENCES}

[1] J. Clerk Maxwell, A Treatise on Electricity and Magnetism, 3rd ed., vol. 2. Oxford: Clarendon, 1892, pp.68-73.

[2] Grady Booch, Robert A. Maksimchuk, Michael W. Engel, Bobbi J. Young, Jim Conallen and Kelli A. Houston: Object-Oriented Analysis and Design with Applications, Third Edition, Addison Wesley Professional publisher, 2007, ISBN-10: 020189551X, ISBN-13: 978-0201895513.

[3] Grady Booch: Object-Oriented Analysis and Design with Applications, Second Edition, Addison-Wesley publisher, 1994, ISBN 0-8053-5340-2.

[4] Watts S. Humphrey: Defect Prevention, In Managing the Software Process, Addison-Wesley publisher, Boston, USA, 1989.
[5] Gerald M. Weinberg: Quality Software Management: Systems Thinking, Dorset House publisher, 1997, ISBN10: 0932633226, ISBN-13: 978-0932633224.

[6] Ram Chillarege: The Marriage of Business Dynamics and Software Engineering, IEEE Software, Vol. 19, No. 6, 2002, pp. 43-49.

[7] Ramaswamy. V, Pushpavathi T. P., Suma. V, "Position Paper: Defect Prediction Approaches for Software Projects Using Genetic Fuzzy Data Mining", 48th Annual Convention, Computer Society of India, Vishakapattanam Chapter, 13th -15th December 2013, Vishakapattanam, India.

[8] Suma. V, Pushpavathi T. P., Ramaswamy. V, "An Approach to Predict Software Project Success by Data Mining Clustering", International Conference on Data Mining Computer, Communication and Mechanical Engineering (ICDCCME) 2012, 21st-22nd December, Bangkok, Thailand.

[9] Ramaswamy. V, Suma. V, Pushpavathi. T. P, “An Approach To Predict Software Project Success By Cascading Clustering And Classification", International Conference on Software Engineering and Mobile Application Modeling and Development (ICSEMA) 2012, 19th - 21st December, Chennai, India.

[10] T. R. Gopalakrishnan Nair, V. Suma, Nitya G. Nair, "Estimation of Characteristics of a Software Team for Implementing Effective Inspection Process Inspection performance Metric", Software Quality Professional Journal, American Society for Quality (ASQ), USA, Vol.13, Issue 2, pp.14-26, 2011.

[11] T. R. Gopalakrishnan Nair, V. Suma, "Defect Management Using Pair Metrics, DI and IPM" CrossTalk, The Journal of Defense Software Engineering, Vol. 24, No 6, 2011, pp.22-27, 2011.

[12] T. R. Gopalakrishnan Nair, V. Suma, "Implementation of Depth of Inspection Metric and Inspection Performance Metric for Quality Management in Software Development Life Cycle" International Journal of Productivity and Quality Management (IJPQM), InderScience Publishers, USA, 2011. 
[13] T. R. Gopalakrishnan Nair, V. Suma, "A Paradigm for Metric Based Inspection Process for Enhancing Defect Management", ACM SIGSOFT Software Engineering Notes, vol. 35, No.3, pp.1, 2010.

[14] T. R. Gopalakrishnan Nair, V. Suma, Pranesh Tiwari, "Significance of Depth of Inspection and Inspection Performance Metrics for Consistent Defect Management in Software Industry", IET Software, December 2012, Volume 6, Issue 6, pp. $524-535$.

[15] T. R. Gopalakrishnan Nair, Suma. V, Pranesh Kumar Tiwari, "Analysis of Test Efficiency during Software Development Process", Submitted, 2nd Annual International Conference on Software Engineering and Applications (SEA 2011), Singapore, 12th -13th December 2011.
[16] Rashmi N, Suma V., "Defect Detection Efficiency of the Combined Approach of Testing", 48th Annual Convention, Computer Society of India, Vishakapattanam Chapter, 13th -15th December 2013, Vishakapattanam, India.

[17] Rashmi N, Suma V., "Defect Detection Efficiency: A Combined approach", International Conference on Emerging Trends Technology and Research (ICETTR2013), 21st -22nd September 2013, Nagpur, Maharashtra, India.

[18] Jef Jacobs, Jan Vol Moll, Rob Kusters, Jos Trienekens and Aarnout Brombacher: Identification of Factors that Influence Defect Injection and Detection in Development of Software Intensive Products, Information and Software Technology Journal, Vol. 49, No. 7, 2007, pp. 774-789. 